\title{
TEORÍA TRANSTEORÉTICA DE CAMBIO DE CONDUCTA: HERRAMIENTA IMPORTANTE EN LA ADOPCIÓN DE ESTILOS DE VIDA ACTIVOS
}

\author{
Dr. Carlos Álvarez \\ Universidad Nacional, Costa Rica \\ calvarez@una.ac.cr
}

RESUMEN

\begin{abstract}
A pesar del culto a la belleza que ha regido en esta sociedad globalizada, y a la importancia de la actividad física en relación con la prevención de las enfermedades cardiodegenerativas (US.DHHS, 1996), el sedentarismo sigue creciendo en la población. ¿Qué estrategias puede usar un profesional en movimiento humano para lograr activar a la población? Es aquí donde surge el modelo transteorético como una opción innovadora que supera con creces las intervenciones biologistas clásicas y cortoplacistas, donde ha prevalecido el centrarse en los cambios momentáneos dados por valores fisiológicos. Esta teoría muestra gran potencial, ya que ofrece los fundamentos teóricos que posibilitan la adquisición de una conducta activa. Este artículo explica los fundamentos teóricos del modelo transteorético, incluyendo la forma de valorar los estadios de cambio y de desarrollar diferentes estrategias para movilizar a la persona, usando los procesos de cambio y los conductuales.
\end{abstract}

PALABRAS CLAVES: Modelo Transteorético, intervención, actitud de cambio, actividad física, nutrición, procesos conductuales, procesos cognitivos, estadios de cambio.

\section{TRANSTHEORETICAL MODEL OF BEHAVIOR CHANGE: AN IMPORTANT TOOL WHEN ADOPTING AN ACTIVE LIFESTYLE}

\author{
ABSTRACT
}

\begin{abstract}
Despite the worship of beauty that has ruled this globalized society and the importance of physical activity for the prevention of cardio-degenerative diseases (US.DHHS, 1996), sedentary behavior continues to increase in the population. What strategies can be used by professionals of human movement to make the population physically active? It is at this point where the Transtheoretical Model appears as an innovative option that greatly surpasses the classic short-term biological interventions, which have focused on momentary changes produced by physiological values. This model has great potential since it offers the theoretical basis that makes the acquisition of an active behavior possible. This article explains the theoretical basis of the transtheoretical model, including the way to assess the stages of change and to develop different strategies to make the person active by using behavioral processes and processes of change.
\end{abstract}

KEY WORDS: Transtheoritical Model, intervention, behavior change, physical activity, nutrition, behavioral processes, cognitive processes, stages of change.

\section{INTRODUCCIÓN}

Un estilo de vida sedentario es un factor de riesgo para una gran cantidad de enfermedades que se convierten más prevalentes con la edad, en ambos sexos. Con respecto al sedentarismo como factor de riesgo para enfermedad coronaria, Caspersen (1987) notó que hay suficiente evidencia para decir que este factor de riesgo se equipara con los otros factores de riesgo primario. Estos hallazgos revelan que individuos que presentan niveles bajos de aptitud física están en mayor 
URL www.una.ac.cr/mhsalud

riesgo de sufrir enfermedad coronaria que el individuo con buena condición aeróbica (Fletcher y otros, 2001). Más aún, se ha señalado que la inactividad física podría ser el más importante de los factores de riesgo debido al hecho de que más personas son inactivas físicamente que las que fuman o sufren de hipertensión arterial o tienen un nivel alto de colesterol de baja densidad (LDL) (U.S.Department of Health and Human Services, 1996).

En las últimas dos décadas, la evidencia científica ha demostrado que la participación regular en actividad física provee importantes aportes en la salud. Como consecuencia, la promoción de la esta se ha reconocido como una prioridad para la salud pública, así es como las organizaciones mundiales en salud, se han pronunciado en el sentido de la importancia en la prevención de la enfermedad crónicodegenerativa y de los beneficios en la promoción de la salud, a través de la actividad física (Pate y otros, 1999).

En años recientes se han realizado esfuerzos significativos para reducir los factores de riesgo modificables de las enfermedades cardiodegenerativas en la población (no transmisibles). Sin embargo, cuando se habla del sedentarismo, como factor de riesgo, pareciera que los avances son reducidos, por lo que los esfuerzos para promocionar la actividad física entre la población sedentaria en Costa Rica se deben redoblar para impactar la salud pública. Varios estudios (Marcus y Owen, 1992; Álvarez, 2001) indican que el uso de modelos teóricos, como el de los estadios de cambio, han demostrado ser de gran impacto a la hora de estructurar intervenciones para cambiar conductas sedentarias. Con el objeto de darle alguna herramienta adicional al profesional en salud y movimiento humano, este artículo presenta el modelo transteorético de cambio de conducta, los fundamentos teóricos del modelo, incluyendo la forma de valorar los estadios de cambio y de desarrollar diferentes estrategias para movilizar a la persona usando los procesos de cambio y los conductuales.

\section{DESARROLLO (Justificación)}

Varios acercamientos están disponibles para motivar a la población en la adopción de conductas de vida activa, sin embargo, algunas de estas teorías psicológicas basan sus supuestos en aspectos mecanicistas, que reducen los cambios a un asunto muy simplista. Las intervenciones mecanicistas para promover la actividad y hábitos apropiados a través del cambio de conducta, como por ejemplo la confección de prescripciones de ejercicio, los acuerdos escritos, contratos de conducta, selección de metas y la toma de decisiones, han estado asociadas con un $10 \%$ o $25 \%$ en el aumento de la actividad física o de la práctica deportiva y es probable que estén asociados a cambios de corta duración (Dishman, 1991). OConnor (1994), sugiere que las intervenciones que han utilizado conceptos biologistas como una receta, no han dado los resultados esperados. Aunque estos estudios e intervenciones han mostrado cambios pasajeros, también se les crítica los diseños de estos estudios o intervenciones. El cuestionamiento fundamental es que se usaron estudios preexperimentales y cuasiexperimentales. Una segunda razón, es que los estudios realizados no han estado basados en modelos teoréticos del cambio de conducta que incluye el conocimiento reciente en la ciencia del cambio de conducta, la ciencia del ejercicio, la ciencia de la nutrición y la de salud pública (Dishman, 1991).

Es un hecho que el éxito de cualquier programa de promoción de la salud debe tener claro las teorías de la conducta, y sus procesos de cambio. Entre mejor se entienda los factores que influencian una conducta deseada y el contexto social en el cuál ocurre, más posibilidades existirán que el diseño de intervención logre impactar las conductas deseadas (Prochaska y otros, 1994). Las teorías de la conducta ofrecen una explicación acerca de la complejidad de los factores que influyen en ella de una forma u otra y en un punto dado en el tiempo. Estas teorías contribuyen al entendimiento de la naturaleza de la conducta deseada que se quiere promocionar y de la indeseable que se quiere reemplazar. 
URL www.una.ac.cr/mhsalud

Uno de los acercamientos tradicionales para controlar conductas inapropiadas ha sido el de proveer información acerca de las consecuencias negativas de la actividad. Así es como, a la hora de tratar de activar un determinado grupo, lo común es dar información de las consecuencias de ser sedentario y recomendar ejercicio desde un punto de vista biologista. Dando recomendaciones de las consecuencias negativas, de las conductas de riesgo que no han disminuido, ni activado a la población. El sedentarismo es un factor de riesgo coronario, que no ha sido modificado por un acercamiento basado en la información. Otro modelo clásico, es de asumir que la persona cambiará su conducta con el hecho de elaborarle un plan basado en los principios de la metodología de la actividad física. Este tipo de intervenciones respetan todos los principios del entrenamiento, usa fórmulas basadas en hallazgos científicos y han sido confeccionadas por profesionales, sin embargo, estos acercamientos se han caracterizado por un porcentaje bajo de adherencia; pero con el afán de darle una solución a los problemas tradicionales de las intervenciones que pretenden activar a la población, se ha escogido la teoría de los estadios de cambio, teorías más significativas en el campo de la modificación de la conducta y la salud. Esta selección obedece al hecho de que los modelos que representa esta teoría han sido aplicados y analizados en una variedad de lugares, tomando en cuenta un análisis de las teorías psicoterapéuticas de avanzada (Prochaska, DiClemente y Norcross, 1992).

Esta teoría se basa en el concepto de cambio para la reconstrucción de las condiciones de salud de la sociedad. Se considera como punto de inicio la premisa de que el cambio no es un evento aislado, sino un proceso y que el proceso de aprendizaje debe respetar las formas naturales de conocer y manejar el entorno del individuo. En el concepto de cambio se tomará el modelo transteorético de cambio de conducta, el cuál considera al cambio como un proceso, en vez de un evento aislado en el tiempo. Este modelo está compuesto por dos dimensiones: las etapas y los procesos de cambio. Las etapas de cambio se refieren a lo temporal, motivacional, y a la constancia del cambio, mientras que los procesos de cambio son aquellas actividades que tienen lugar entre las etapas. Con esto en mente se parte del hecho de poder clasificar a las personas dentro de uno de los estadios de cambio (Prochaska y otros, 1994).

Este modelo, transteorético de cambio de conducta, tiene sus orígenes en estudios de conductas adictivas como el abuso del alcohol, fumado, la obesidad, y el abuso de drogas (O'Connor, 1994). Prochaska y Bess (1994) establecen que el cambio es ni más ni menos que un proceso, en lugar de ser interpretado como un evento aislado. Este es un acercamiento con gran potencial para la promoción de la actividad física y de la buena alimentación, ya que esto posibilita a los educadores físicos o profesionales en salud a enfocar el problema de la adherencia a la actividad física que promueva los hábitos individuales de esta. Esta teoría tiene dos dimensiones: estadios y procesos de cambio. El modelo propone que las personas hacen los cambios de conducta en etapas y que ellos se mueven en forma secuencial a través de estadios mientras experimentan diferentes procesos de cambio (DiClemente y otros, 1991). Estos estadios representan una dimensión temporal, durante la cual el cambio puede ocurrir, mientras que los procesos de cambio son estrategias individuales usadas conforme los individuos progresan a través de los estadios de cambio. En otras palabras, los procesos de cambio son aquellas actividades y eventos que conducen a conductas exitosas en el cambio. En este modelo, los clientes pueden ser clasificados en alguno de los cinco estadios, según se puede observar en la figura dibujo 1 (Cardinal y otros, 1998). 


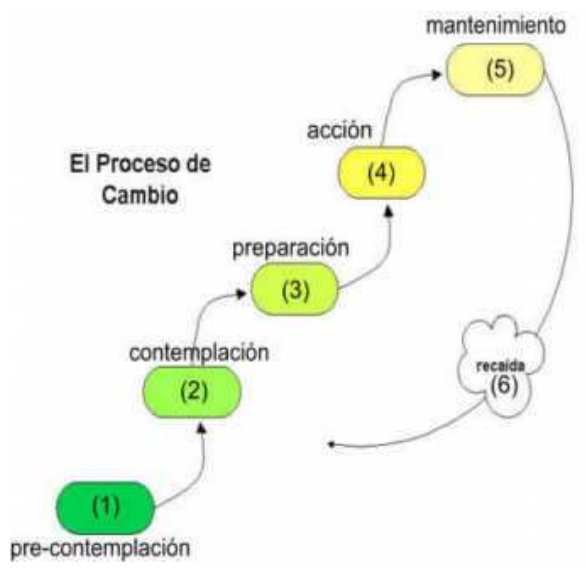

De "Application of the transtheoretical model of behavior change to preadolescents physical activity and exercise behavior," por B. Cardinal, K. Engels y W. Zhu, 1998, Pediatric Exercise Science. 10, 69.

\section{ESTADIOS DE CAMBIO}

El primer estadio de cambio es el de precontemplación, que es el estadio en el cuál no hay intención para el cambio de conducta o deseo de adoptar un estilo de vida activo. Para este O’Connor (1994) recomienda que para el proceso de intervención se utilicen como elementos básicos, el preguntarle al individuo la razón por la que no hace actividad física o por lo que no se alimenta apropiadamente. También, se propone proveer información acerca de la importancia de la actividad física y la buena alimentación, incluyendo los beneficios de la misma y los riesgos de una vida sedentaria. El objeto de este estadio es el de facilitarle al individuo el espacio para que piense en los riesgos de una vida sedentaria y de los beneficios de una vida activa. Algunas estrategias que se pueden implementar están: pedirle al cliente que lleve un diario que incluya entrevistas a personas activas que sean modelos para el sujeto, confección de historias que incluyan personajes que son activos, observar fábulas y determinar el grado de actividad física de los personajes, ver videos que muestren modelos apropiados, realizar pequeñas obras de teatro o mimo que realcen los beneficios de una vida activa, cocinar recetas saludables, propiciar actividades físicas en los recesos (juegos, mecate, concursos, etc.) (Álvarez, 2001). En este estadio es indispensable que los clientes retomen el disfrute de las actividades, a través de la actividad física.

Un segundo estadio es el de contemplación, en el cuál la persona piensa en la posibilidad de ser activo físicamente en un futuro cercano. Una vez que un cliente se ha clasificado en este estadio, el profesor podrá intervenirlo, con el objeto de que pueda avanzar a otro nivel o estadio. Para tal efecto Marcus y Forsyth (2003) recomiendan que aumente el conocimiento del individuo sobre el tema y las oportunidades para que pueda involucrarse en actividades que correspondan en el nivel de destreza de él. Además, se recomienda seguir con las actividades de la fase anterior y con la enseñanza de nuevas destrezas físicas y alimenticias que los faculte para emprender nuevas aventuras. En este momento es apropiado visualizar formas en que ellos pueden ser activos y que comprendan que no solo haciendo deporte se va a llenar los requisitos para una buena salud, sino también por medio del ejercicio y de la actividad física (Corbin y otros, 1996). 
URL www.una.ac.cr/mhsalud

En este punto del modelo, se dice que si la persona interioriza las estrategias anteriores estaría en el estadio de preparación. Estaría decidiendo, preparándose o comenzando a realizar pequeños cambios. Este es un punto crítico en los estadios de cambio, el cuál requiere de un aumento o eliminación de las barreras que impiden que la persona siga cambiando. Además, se requiere que el promotor o docente le ayude a proponerse metas realistas. La estrategia conlleva la sustitución de conductas pasivas por otras que conduzcan a activarse. Punto clave es el estímulo constante, basado en los logros y no en aspectos generales (Cardinal y otros, 1998).

Algunos autores, como por ejemplo Marcus y otros (1992), sostienen que además de los estadios anteriores, es necesario incluir una fase de acción, que es cuando el individuo modifica su conducta, experiencias y ambiente para iniciar nuevos patrones de movimiento y alimentación. El periodo de inicio y los seis meses se consideran críticos, ya que es en este momento cuando la persona es más propensa a abandonar las nuevas conductas (Dishman, 1988). El cambio realizado en sus patrones de movimiento o alimentación, requiere de acompañamiento y refuerzos permanentes, los cuales deben ser basados en los logros y no en comentarios generales de éxito. Además, en este punto se facilita mucho la permanencia del sujeto en esta etapa, si se desarrolla una cadena de apoyo, como por ejemplo la familia, así es como los padres podrían involucrarse con sus hijos durante la semana en alguna actividad física, que no solo de soporte a los cambios de sus hijos, pero también a un cambio en sus patrones de movimiento. Lo mismo podría hacer los padres, si el alumno decide incluir dentro su alimentación la ingesta de frutas cotidianamente (Wright y otros, 2000).

La última etapa de este modelo es la de mantenimiento, que es cuando el individuo ya lleva más de seis meses con los cambios de conducta. Un sujeto que se encuentra en este estadio, se le debe incentivar para que explore nuevas alternativas de actividad física o para que involucre nuevas formas de alimentación, que le ofrezcan mayor variedad. En este momento del proceso, surge la necesidad de focalizar obstáculos, que impiden o minimizan las posibilidades de éxito (Cardinal y otros, 1998).

\section{PROCESOS DE CAMBIO}

Dentro de la dinámica de la teoría transteorética los estadios ayudan a situar al cliente en un punto determinado sin embargo, poco se lograría sabiendo esto y desconociendo las estrategias que se podrían implementar para propiciar que el sujeto escale posiciones y así se logre llegar hasta el estadio apropiado. Los procesos de cambio son las actividades que propician que el individuo se movilice hacia un nuevo estadio (OConnor, 1994). Prochaska, DiClemente y Norcross (1992) identifican diez procesos de cambio. Estos serán descritos someramente, siguiendo el aporte que hace Marcus y otros (1992).

1. Elevación de la conciencia: tiene que ver con los esfuerzos individuales en la búsqueda de información y su correspondiente entendimiento en relación con determinado problema.

2. Reevaluación del ambiente: es una evaluación por parte del sujeto del problema y como este incide sobre el ambiente social y físico.

3. Alivio dramático: contempla los aspectos afectivos del cambio, frecuentemente incluye experiencias emocionales relacionadas al problema de conducta.

4. Autoevaluación

5. Liberación social: conciencia, disponibilidad y aceptación por el sujeto de alternativas.

6. Contra-condicionamiento: es la sustitución de conductas alternativas al problema.

7. Relaciones de ayuda: es la utilización de apoyo por otros en el intento de cambio.

8. Administración de los refuerzos: cambia la estructura que le da sostén al problema.

9. Autoliberación: es la escogencia y compromiso del individuo para cambiar la conducta, incluyendo la idea de que puede cambiar.

10. Control del estímulo: es el control de situaciones y otras causas que inician la conducta no deseada. 
Otros autores como Marcus y Forsyth (2003), presentan procesos cognitivos y conductuales, los cuales son más aplicables en un contexto móvil y real. Si se presta atención a los procesos que se presentan a continuación, se podrá notar que el camino es claro a la hora de intervenir a un sujeto, grupo comunal, industrial o escolar sin embargo, es necesario saber en que momento estos procesos pueden ser más efectivos. Para este caso, en uno de los apartados de este artículo se mostrará en que estadio se usa cuál proceso en mayor medida.

Procesos cognitivos:

Aumentar conocimiento

Ser conciente de los riesgos de la conducta sedentaria

Analizar el impacto de la conducta en la vida de otros

Entender los beneficios de una conducta activa

Aumentar las posibilidades de conductas saludables

Procesos conductuales:

Sustituir conductas sedentarias por activas

Incluir soporte social

Premiarse por pequeños grandes logros

Compromisos personales (contratos)

Recordatorios permanentes (ropa deportiva en el trabajo, camiseta extra para caminar durante el café)

Prochaska y DiClemente (1985), proponen que la aplicación de las estrategias depende del estadio de cambio en que se encuentre el sujeto. Esto sugiere que la intervención que tome en cuenta lo anterior, aumentará la efectividad de la intervención. Basado en investigación seccional cruzada Prochaska y DiClemente (1985) proponen que los educadores físicos o de salud enfaticen procesos específicos durante los estadios específicos de cambio.

Uno de los puntos atractivos de este modelo es que puede ayudar a los educadores en salud a tener un mejor entendimiento de sus clientes (sujetos). Por ejemplo, si los facilitadores pueden reconocer que un número determinado de sujetos se encuentra en un estadio determinado, ellos pueden implementar estrategias que propicien un cambio. Esto también es importante debido, como se mencionó previamente, que los patrones de comportamiento se establecen temprano en la vida (Wright, Patterson y Cardinal, 2000).

Dependiendo en el estadio en el que el cliente se encuentre, así serán las estrategias que se podrán implementar para aumentar la actividad o para mejorar la calidad de la alimentación (Marcus y Forsith, 2003).

Con los estadios se explica cuando las personas cambian, y con los procesos se explica como cambian, debido a esto antes de pasar a la parte aplicativa, se presentarán los procesos cognitivos y los conductuales. Según Marcus y Forsyth (2003) todos los procesos son importantes y pueden ser usados según el estadio de cambio sin embargo, se recomienda que se apliquen como se presentan en este documento.

\section{ESTRATEGIAS PARA CADA UNA DE LAS TEORÍAS DE CAMBIO}

El paso inicial cuando se interviene una persona o grupo, es el de establecer el estadio de cambio en que se encuentra cada uno de los sujetos que tomarán parte en el programa, esto con el fin de decidir las estrategias particulares que se deberán utilizar en cada caso (Cardinal et al, 1998) A continuación encontrarán uno de los cuestionarios, que se usan para clasificar a los participantes de un programa que pretenda cambios de conducta hacia estilos de vida más activos. Mantenga en mente que este cuestionario es uno de los más simples, por lo que se 
URL www.una.ac.cr/mhsalud

recomienda ampliamente. Una vez que la persona está situada en un estadio de cambio, diríjase al estadio correspondiente y utilice alguna de las estrategias correspondientes.

\section{CUESTIONARIO ACTITUD DE CAMBIO}

Seguidamente, las siguientes oraciones ayudarán a entender su nivel de actividad física. Por favor lea la oración y marque el número de la oración que mejor representa su estado actual de actividad física o su interés en actividad física (Cardinal, Engels y Zhu, 1998).

$1=$ precontemplación $2=$ contemplación $3=$ preparación $4=$ activación $5=$ mantenimiento

1. Actualmente no soy físicamente activo o activa, y no estoy pensando en cambiar.

2. Estoy pensando en realizar actividad física en los próximos días.

3. Soy activo, pero no regularmente.

4. Actualmente soy activo, pero inicie la actividad física hace menos de 6 meses.

5. Actualmente soy activo o activa y llevo 6 meses de hacerlo

Ahora que usted o el sujeto que usted está aconsejando, conocen el estadio en que se encuentra, localice en los apartados siguientes las estrategias que se recomiendan para el estadio respectivo y aplíquelas.

\section{ESTRATEGIAS DE APLICACIÓN DE LA ETAPA DE PRECONTEMPLACIÓN}

En esta etapa es básico usar el proceso cognitivo de conocimiento y el de establecer algunas metas que conduzcan a conductas elementales de movimiento, como por ejemplo: segundos de movimiento en camino al trabajo. No presione una activación prematura en esta etapa, ya que el sujeto puede desertar al encontrar mucha presión o al considerar que el activarse no vale la pena. La misión en esta etapa es desarrollar en el cliente el deseo por realizar actividad física. Algunas de las estrategias siguientes han dado excelente resultado en diferentes poblaciones; sin embargo, sea cauto y use algunas de ellas, con el propósito de que no saturen al individuo (Marcus y Forsyth, 2003).

1. Aumentar confianza en habilidades para cambio.

2. Mencione riesgos de ser sedentario y los beneficios.

3. Motivar para realizar planes de cambio y hoja de cambio.

4. Discuta y haga que el sujeto resuelva las barreras para la activación.

5. Recomiende redes de apoyo (familiares, amigos y grupos sociales).

6. Discuta posibles actividades por realizar y evalué barreras para realizarlo.

7. Clarifique ambivalencia entre lo que logrará y lo que la persona espera.

8. Desarrolle un diario actividades diarias.

9. Observe su show favorito y calcule la cantidad de movimiento que realizan los participantes.

10. Establezca las diferencias entre actividad física y ejercicio.

11. Mencione estrategias que usan las personas para evitar la

12. actividad física.

13. Mencione actividades que pueden ser adoptadas.

Una sesión grupal o charla comunal puede ser usada para impactar a las personas que están en la etapa de repudio hacia la actividad física. Adicionalmente, no espere que la persona salte a pensar en la posibilidad de hacer actividad física. Resuelva primero las causas de la aversión al movimiento, las cuales pueden ser: malas experiencias, falta de conocimiento, vergüenza de usar ropa deportiva, falta de tiempo. Muchas de las razones para no activarse terminan siendo un círculo de excusas que frustran a ambos. Pare el círculo y trate la técnica de resolución de 


\section{ESTRATEGIAS DE APLICACIÓN DE LA ETAPA DE CONTEMPLACIÓN}

Al igual que el estadio de precontemplación, el estadio de contemplación es inestable y requiere que el profesional en salud no asuma gran estabilidad del participante. Por ser esta etapa o estadio, esencial en el futuro de la intervención, se recomienda iniciar a estructurar la aplicación de los procesos de cambio de conocimiento y de los riesgos que acarrea una conducta sedentaria, igualmente el proceso conductual de actividades alternativas es apropiado recomendarlo. Tenga cuidado en no involucrar a la persona en ejercicio, sino más bien, en buscar posibilidades dentro del horario del sujeto de incrementar algunos minutos de actividad física. Ponga una fecha para el inicio de los incrementos en actividad física y decidan metas a corto y mediano plazo (Wrigth, Patterson y Cardinal, 2000).

En este estadio todavía pueden persistir obstáculos para activarse, por lo que el profesional en salud debe propiciar la resolución de problemas en una dinámica de uno a la vez. Una meta manejable en este estadio es, primero que la persona comprenda las diferencias entre las diferentes componentes del movimiento humano, para poder incluir en la rutina de la persona cinco minutos de actividad física en un espacio viable.

\section{ESTRATEGIAS DE APLICACIÓN PREPARACIÓN}

En este punto, el individuo está sumergido en la espiral de cambio, por lo que se requiere que el individuo que está realizando actividad física en forma esporádica, asuma los retos y compromisos de una persona que ya ha avanzado en la adopción de los procesos cognitivos, esto sin olvidar los conductuales. En este estadio los procesos se entrecruzan, para lograr lo mejor de cada una de las estrategias. Se le puede sugerir a la persona algunas de las estrategias siguientes, con el afán de que el deseo de realizar actividad física, sea materializado en una conducta estable (Marcus y Forsyth, 2003).

- Fijar metas y firmar contrato basándose en realidad (estrategias abandono).

- Reforzar pequeños cambios.

- No recomiende cambios generales.

- No se refiera a los pequeños cambios como insignificantes.

- Unirse a grupos y llevar un record de actividad física.

- Evaluar metas y decidir cómo se puede aumentar la cantidad de actividad física.

- Mantenga metas apegadas a la realidad.

- Decida en qué momento del día es más energético.

- Trate de incluir amigos o familiares en sus actividades.

- Planee con la familia actividades activas en el fin de semana.

- Es tiempo de premiarse, elija cosas con las cuales puede incentivar los logros obtenidos.

- Introduzca estiramiento y relajamiento en su rutina.

- Esté seguro que al realizar la actividad física pueda hablar confortablemente.

- Cuando determine una conducta sedentaria, escoja paralelamente la opuesta.

Tradicionalmente, en las intervenciones conocidas, se han utilizado refuerzos materiales para mantener conductas de movimiento (Marcus y Forsyth, 2003); sin embargo, estas estrategias no han desembocado en conductas permanentes, por el contrario, pierden su efectividad con el tiempo. Lo que si se ha determinado como una estrategia efectiva es el uso de un puntaje por meta lograda, esto con el fin de ir interiorizando el manejo de los procesos y de probar efectividad de los mismos. 


\section{ESTRATEGIAS DE APLICACIÓN EN LA ETAPA DE ACCIÓN}

Aunque pareciera que se pueden tirar las campanas al aire, no baje la guardia, ya que el participante está sujeto a que abandone el estilo activo de vida, si no encuentra apoyo en el entorno. En este estadio se requiere que se establezca una rutina que será cotejada en una hoja para tal efecto y con valoraciones físicas, con el afán de que ayuden a valorar los cambios del participante, aumentar la motivación y para realizarle ajustes en la rutina de actividad física.

1. Ayudar con retroalimentación (mediciones físicas ayudan).

2. Cooperar con capacitación continua.

3. Programas educativos, mezclados con actividad física novedosa.

4. Proveer materiales autodigeribles.

5. Trazarse metas que impliquen retos grupales.

6. Siempre buscar la ayuda grupal.

7. Se pretende formar un anillo de apoyo que se involucre y conozca las metas.

8. Escoja refuerzos que mantengan la motivación alta.

9. Estimule a algún amigo a unirse en pequeñas actividades recreativas.

10. Organice una caminata por placer con sus amigos.

11. Determine si hay alguna barrera que le impide algunas veces realizar la actividad.

12. Confeccione una lista de los beneficios que ha logrado hasta ahora.

\section{ESTRATEGIAS ADICIONALES}

Estrategia 1: recuerde en seleccionar metas a corto y largo plazo. por ejemplo: si su deseo es participar en una caminata para la salud de 10 kilómetros dentro de 3 meses y usted solo camina 4 kilómetros, es hora de que piense si puede cumplir la meta y de cómo piensa incrementar el tiempo para el logro de la misma. Siempre establezca metas realistas.

Estrategia 2: es tiempo de unir a su actividad a otras personas y de asistir a charlas en estilos de vida saludables. Invite a familiares y amigos a acompañarlo. Explíqueles también de los planes de ser más saludable y solicíteles cooperación.

En esta fase el participante ya realiza la actividad física necesaria para obtener beneficios para la salud, por lo que es tiempo de reevaluar las metas e introducir algún tipo de medición que ayude a motivar los logros a partir de este momento (frecuencia cardiaca, minutos totales caminados, pasos dados).

\section{ESTRATEGIAS DE APLICACIÓN EN LA ETAPA DE MANTENIMIENTO}

Estar en esta etapa significa que la persona ha interiorizado los procesos cognitivos y los conductuales, lo que le otorga la posibilidad de aplicar los procesos aprendidos y las respectivas estrategias; sin embargo, la supervisión del profesional se hace necesaria para seguir reforzando lo aprendido (Cardinal y otros, 1998). Es básico que el participante anticipe dificultades ambientales, las cuales podrían hacer que el individuo retroceda en la espiral de cambio.

1. Apoyo social.

2. Anticipar dificultades y desarrollar plan.

3. Recomendar otras actividades si el cliente está motivado.

4. No asuma la permanencia en esta etapa.

5. No se desmotive si hay abandono de la actividad.

6. Promueva actividad física entre los conocidos

Estrategia adicional 1. Si tiene que salir de viaje o va de paseo anticipe actividades físicas que 
Estrategia 2. Si por motivo de una enfermedad leve detiene la actividad física, aproveche el tiempo leyendo algún tipo de literatura sobre estilos de vida activos.

Estrategia 3.Localice grupos comunitarios de actividad física que le ofrezcan nuevas actividades.

Estrategia 4. Mantenga metas anuales de participación en eventos recreativos. Comparta con otros los logros y motívelos a cambiar poco a poco.

Este es momento de verificar los procesos cognitivos y conductuales que se están manejando y de garantizar el disfrute de la actividad. Además, considere importante después de seis meses de actividad física, desarrollar estrategias de contingencia, en caso que haya un abandono, viaje, cambio de residencia u otro imprevisto que amenace los logros.

A través de cada uno de los estadios presentados se han sugerido las estrategias o procesos necesarios para movilizar a los sujetos de un estadio a otro. Cuando la gente se encuentra en el estadio 2, ellos están usando en mayor medida procesos cognitivos, por el contrario, si los sujetos están en el estadio 4, ellos estarán utilizando mayor cantidad de procesos conductuales. Lo anterior, no quita que el facilitador en salud pueda recurrir a usar ambos tipos de procesos especialmente en las etapas superiores o cuando la persona abandona la espiral de cambio. El facilitador debe contemplar que los sujetos pueden avanzar un estadio o descenderlo, sin que ponga en peligro el proceso de cambio. Más bien, se espera que el sujeto tenga avances de un estadio o retrocesos como parte normal del proceso. Lo que no es aconsejable es ver a un sujeto avanzar tres estadios en una semana. Si lo anterior sucediera, que es muy frecuente, especialmente en personas que no tienen conciencia del tiempo que llevan siendo sedentarios, lo que se recomienda es que se permita al sujeto cierta libertad, mientras el mediador ejecuta el proceso en el orden sugerido.

Muchos de los mediadores en salud, no toman en cuenta una etapa sexta, que es la de abandono; sin embargo, el promotor en movimiento humano, conoce lo fácil de tener una recaída a través de las etapas de la vida, y por que no muchas recaídas. Conscientes de este proceso, es válido mantener un control de los procesos adoptados, con el afán de reforzar y verificar la interiorización de los mismos.

\section{CONCLUSIÓN}

El modelo transteorético, con sus estadios de cambio, ha demostrado ser de gran impacto a la hora de estructurar intervenciones para cambiar conductas sedentarias. Los fundamentos teóricos del modelo, incluyendo la forma de valorar los estadios de cambio y de desarrollar diferentes estrategias para movilizar a la persona de un estadio a otro, podrán ser implementados siguiendo lo presentado en este artículo.

El potencial del modelo transteorético para asistir a los profesionales en movimiento y la salud, en la tarea de activar a la población es significativo, especialmente cuando se aplican apropiadamente los procesos. Este dominio garantiza el uso pertinente de las estrategias que conducirán a movilizar a la persona a un nuevo estadio.

\section{REFERENCIAS}

Álvarez, C. (2001). Teoría transteorética: aplicación en estudiantes de secundaria. Manuscrito no publicado. 
URL www.una.ac.cr/mhsalud

Cardinal, B. (1997). Construct validity of stages of change for exercise behavior. American Journal of Health Promotion. 12, 68-74.

Cardinal, B., Engels, K., and Zhu, W. (1998). Application of the transtheoretical model of behavior change to preadolescents physical activity and exercise behavior. Pediatric Exercise Science, 10, 69-80.

Caspersen, A. (1987). Physical inactivity and coronary heart disease. Physician and Sport Medicine, 15, 43-24.

Corbin, C. y Pancrazi, R. (1996). How much physical activity is enough? JOPERD: 67, 33-39.

DiClemente, C., Prochaska, J., Fairhurst, S., Velicer, W., Velasquez, M. and Rossi, J. (1991). The process of smoking cessation: an analysis of precontemplation, contemplation, and preparation stages of change. Journal of Consulting and Clinical Psychology, 59, 295305.

Dishman, R. (1988). Exercise Adherence. Champaign, IL: Human Kinetics.

Dishman, R. (1991). Increasing and maintaining exercise and physical activity. Behavior Theory, 22, 345-378.

Fletcher, F., Gary, J. Balady, Ezra A. Amsterdam, Bernard Chaitman, Robert Eckel, Jerome Fleg, Victor F. Froelicher, Arthur S. Leon, Ileana L. Piña, Roxanne Rodney, Denise A. Simons-Morton, Mark A. Williams, Terry Bazzarre, (2001). Exercise standards for testing and training. Circulation, 104, 1694.

Marcus, B. and Forsyth, F. (2003). Motivating People to be Physically Active. Nueva York: Human Kinetics.

Marcus, B., Selby, V., Niaura, R., and Rossi, J. (1992). Self-efficacy and the stages for exercise behavior change. Research Quarterly for Exercise and Sport, 60 (1), 66.

Marcus, B. and Owen, N. (1992). Motivational readiness, self-efficacy and decision-making for exercise. Journal of Applied Social Psychology., 22, 3-16.

OConnor, Ml. (1994). Exercise promotion in physical education: Application of the Transtheoretical Model. Journal of Teaching in Physical Education, 14, 2-12.

Pate, C., Trost, S., Dpwda, M.,Ott, A., Ward, D. and Saunder, R. (1999). Tracking of physical activity, physical inactivity, and health-related physical fitness in rural youth. Pediatric Exercise Science, 11, 364-376.

Prochaska, J. and Bess, M. (1994). The transtheoretical model: applications to exercise. Advances in exercise adherence. Dishman, R. (edit). Nueva York: Human Kinetics Publishers.

Prochaska, J., DiClemente, C. and Norcross, J. (1992). In search of how people change. American Psychologist, 47, 1102-1114.

Prochaska, J. and DiClemente, C. (1985). Coping and Substance Use. New York: Academic Press. 


\section{MHSalud}

URL www.una.ac.cr/mhsalud

U.S.Department of Health and Human Services. (1996). Physical activity and health - A report of the Surgeon General. Atlanta, GA: Centers for Disease Control Printing Office.

Wrigth, M., Patterson, D. and Cardinal, B. (2000). Increasing children physical activity. JOPERD, 71, 26-29.

Fecha de recepción del artículo: 11 de octubre del 2007.

Fecha de aceptación del artículo: 02 de abril del 2008.

Fecha de publicación del artículo: 31 de julio del 2008. 